\title{
A Note on Divisibility of the Number of Matchings of a Family of Graphs
}

\author{
Kyung-Won Hwang \\ General Education Department, Kookmin University \\ 861-1 Jeongneung-dong Seongbuk-gu 136-702, South Korea \\ khwang7@kookmin.ac.kr \\ Naeem N. Sheikh \\ Department of Mathematics and Statistics, Miami University \\ Oxford, $\mathrm{OH} 45056$ \\ sheikhnn@muohio.edu \\ Stephen G. Hartke* \\ Department of Mathematics, University of Nebraska-Lincoln \\ 203 Avery Hall, P.O. Box 880130, Lincoln, NE 68588-0130, USA \\ shartke2@math.unl.edu
}

Submitted: May 8, 2006; Accepted: Mar 9, 2007; Published: Mar 20, 2009

Mathematics Subject Classification: 05A15

\begin{abstract}
For a certain graph obtained by adding extra vertices and edges to the triangular lattice graph, Propp conjectured that the number of perfect matchings of such a graph is always divisible by 3 . In this note we prove this conjecture.
\end{abstract}

In a graph $G$, a matching is a set of edges such that no two edges are incident to each other. A matching in a graph is called perfect if every vertex is incident with an edge of the matching. In particular, graphs on an odd number of vertices have no perfect matchings. Many different problems of matchings have been studied: existence, construction, and enumeration are three big categories of problems involving matchings.

For a somewhat detailed history of the task of enumerating perfect matchings of different graphs, we refer the reader to the introduction section in Propp [1]. Researchers

\footnotetext{
${ }^{*}$ Research partially supported by a Maude Hammond Fling Faculty Research Fellowship from the University of Nebraska Research Council.
} 


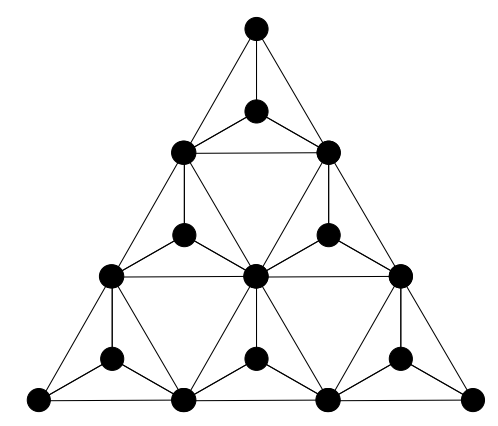

Figure 1: The graph $G_{3}$.

have focused special attention on this problem when the graphs in question are planar, have a repeating pattern and/or have a geometric description. Some important families of graphs in this regard are two-dimensional grids [2, 3], Aztec diamonds [4], and honeycomb graphs [5]. A reason this has attracted considerable interest, from both mathematicians and specialists in other areas, is that a matching can represent a phenomenon in a number of settings. For example, a perfect matching in a two dimensional lattice of molecules could represent the way atoms pair up under the forces of hydrogen bonding.

When finding a formula for the number of perfect matchings of a graph has seemed hard, researchers have focused on the questions of divisibility of the number of perfect matchings by powers of various small primes. Sometimes there is a compact formula for the number of matchings, but it involves irrational numbers (for example, for the number of matchings of a rectangular grid-graph as found in [2]) and thus information about divisibility by particular integers is hard to extract.

In this paper, we consider the planar graph $G_{n}$ obtained from the triangular lattice with $n$ rows of triangles by adding extra vertices and edges in the triangular faces pointing "upwards", as shown in Figure 1. James Propp [1] conjectured that the number of perfect matchings of $G_{n}$ is always a multiple of 3 . Let $M\left(G_{n}\right)$ denote the number of perfect matchings of $G_{n}$. We prove Propp's conjecture, and in fact prove that $3^{\frac{n+1}{2}}$ divides $M\left(G_{n}\right)$. We also prove that there is an extra factor of 3 when $n$ is 0 mod 3 . Note that for even $n$, the number of vertices in $G_{n}$ is odd and hence $M\left(G_{n}\right)=0$ for such a graph. Doug Lepro solved the same problem in his unfinished Ph.D. thesis - a work which is unpublished and dates back more than six years. We also note that our method of proof is somewhat similar to the method employed by Pachter in [6] in which he proves divisibility by powers of 2 for the number of matchings of the square grid graph.

Theorem 1 For $n$ odd, the number of perfect matchings of $G_{n}$ is a multiple of $3^{\frac{n+1}{2}}$.

Proof. We use the term block to refer to a triangular face of the triangular lattice in which there is an extra vertex and three extra edges. Thus, $G_{n}$ can be seen as a triangular arrangement of edge-disjoint blocks some of which share vertices with others. Given a 

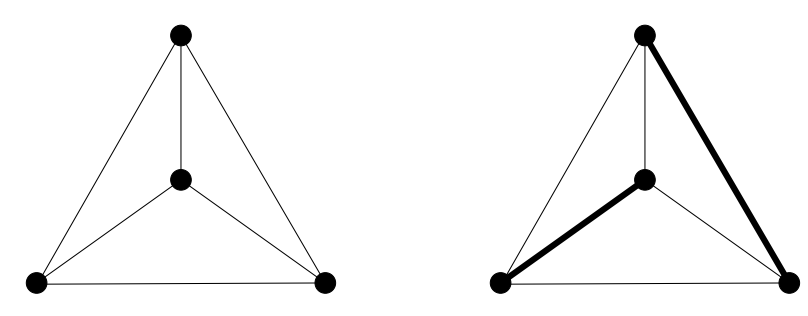

Figure 2: Examples of blocks. The block on the right contains a matching of two edges (shown in bold) and hence is internally satisfied.

perfect matching of $G_{n}$, we call a particular block internally satisfied if it contains two edges of the perfect matching (i.e. the four vertices in the block are matched to vertices within the block). Figure 2 illustrates the concept of a block and an internally satisfied block. First we note that a block itself can be internally satisfied in 3 different ways (as the reader may have observed, a block is isomorphic to the graph $K_{4}$, and $K_{4}$ on labeled vertices has 3 perfect matchings). The main observation is that when a block is internally satisfied in a perfect matching, then changing the pairing within the block to either of the other two ways still gives us a perfect matching of the overall graph.

Counting the number of vertices in $G_{n}$ by rows from top to bottom, we see that the total number of vertices in $G_{n}$ is $2(1+2+\ldots+n)+n+1=n(n+1)+n+1$. Therefore, the number of edges in a perfect matching of $G_{n}$ is $\frac{1}{2} n(n+1)+\frac{n+1}{2}$. The number of blocks in $G_{n}$ is $1+2+\ldots+n=\frac{1}{2} n(n+1)$.

Now, note that in any perfect matching of $G_{n}$, each block must contain at least one edge of the matching, because the center vertex in each block can be paired only with one of the other 3 vertices of the block. Furthermore, each block can have at most two edges of any perfect matching (since each block has 4 vertices). Since the number of edges in any perfect matching is $\frac{n+1}{2}$ more than the number of blocks, we have that in any perfect matching exactly $\frac{n+1}{2}$ blocks have two edges. In other words, in any perfect matching, exactly $\frac{n+1}{2}$ blocks will be internally satisfied.

Now, consider the set of all perfect matchings of $G_{n}$. Define a relation $R$ on this set as follows: two perfect matchings have the relation $R$ if and only if they have the same set of $\frac{n+1}{2}$ internally satisfied blocks and their edges outside these blocks are the same. It is easily seen that this binary relation is an equivalence relation on the set of all perfect matchings of $G_{n}$. Now, we claim that each of the equivalence classes under $R$ has cardinality $3^{\frac{n+1}{2}}$. Thus, the total number of perfect matchings is a multiple of $3^{\frac{n+1}{2}}$.

It remains only to prove the claim that each equivalence class has cardinality $3^{\frac{n+1}{2}}$. This follows from our earlier observation: each of the internally satisfied blocks can be rematched in any of the 3 ways without disturbing the remaining matching. Since each of the $\frac{n+1}{2}$ blocks can be rematched independently in three different ways, it follows that each equivalence class has cardinality $3^{\frac{n+1}{2}}$. 
Theorem 2 For $n$ odd, and $n$ congruent to $0 \bmod 3$, the number of perfect matchings of $G_{n}$ is a multiple of $3^{\frac{n+3}{2}}$.

Proof. We start with the set of equivalence classes under the relation $R$ defined above and construct an equivalence relation $T$ on this set. First note that a given equivalence class under $R$ is determined by specifying the $\frac{n+1}{2}$ internally satisfied blocks and by specifying the edges in the matching that are outside the internally satisfied blocks. We use the term pattern to mean the specification for an equivalence class under $R$ (we visualize this by coloring the blocks that are internally satisfied with gray color). We say that two such patterns are related under the relation $T$ if one can be obtained from the other by rotation through 0,120 or 240 degrees. Note that $T$ is an equivalence relation.

We now show that each equivalence class under $T$ has cardinality 3 . In other words, there is no pattern that is fixed under rotation by 120 or 240 degrees. Observe that when $n$ is $0 \bmod 3$ and $n$ is an odd number, there is no centrally-located block in $G_{n}$. If a pattern were to be fixed under rotation by 120 or 240 degrees, it would have to have the number of internally-satisfied blocks be a number that is a multiple of 3 . However, $\frac{n+1}{2}$ is not divisible by 3 if $n$ is $0 \bmod 3$. Thus no pattern is fixed under rotation by 120 or 240 degrees. Hence, every equivalence class under $T$ has cardinality 3. Given that each pattern is itself an equivalence class of size $3^{\frac{n+1}{2}}$ and the number of patterns is divisible by 3 , we have that $3 \times 3^{\frac{n+1}{2}}=3^{\frac{n+3}{2}}$ divides $M\left(G_{n}\right)$.

\section{Acknowledgements}

We thank Professor Alexandr Kostochka for useful comments. We also thank the anonymous referee for improvements, and for pointing out Doug Lepro's work and the work by Pachter.

\section{References}

[1] J. Propp, Enumeration of Matchings: Problems and Progress, New Perspectives in Geometric Combinatorics, MSRI Publications, 38, (1999) 255-291.

[2] P. W. Kasteleyn, The statistics of dimers on a lattice I: The number of dimer arrangements on a quadratic lattice, Physica, 27, (1961), 1209-1225.

[3] H. N. V. Temperley and M. E. Fisher, Dimer problem in statistical mechanics - an exact result, Phil. Mag., 6, (1961), 1061-1063.

[4] N. Elkies, G. Kuperberg, M. Larsen, and J. Propp, Alternating sign matrices and domino tilings, J. Algebraic Combin., 1, (1992), 111-132 and 219-234.

[5] G. Kuperberg, Symmetries of plane partitions and the permanent-determinant method, J. Combin. Theory Ser. A, 68:1, (1994), 115-151.

[6] L. Pachter, Combinatorial approaches and conjectures for 2-divisibility problems concerning domino tilings of polyominoes, Electron. J. Combin., 4 (1), (1997), R29. 